\title{
Modelación de los stocks de carbono del suelo y las emisiones de dióxido de carbono (GEI) en sistemas productivos de la Altillanura Plana
}

\author{
Modeling soil carbon stocks and carbon dioxide emissions \\ (GHG) in production systems of Plain Altillanura
}

\section{Modelagem dos estoques de carbono do solo e as emissões de dióxido de carbono (GEE) em sistemas de produção da Altillanura Plana}

\author{
Amanda Silva-Parra ${ }^{1}$ \\ 1 Ingeniera Agrónoma, MSc, PhD, Grupo de Investigación en Sistemas Agrícolas y Forestales ISAF, Facultad de Ciencias \\ Agropecuarias y Recursos Naturales, Universidad de los Llanos, Villavicencio, Colombia. \\ Email: asilvap@unillanos.edu.co
}

Recibido: mayo 31 de 2016

Aceptado: octubre 9 de 2018

\begin{abstract}
Resumen
Los distintos manejos de los suelos afectan las existencias de C del suelo. El análisis multivariado de la modelación de los stocks de $\mathrm{C}$ del suelo $\left(\mathrm{SOC}_{0-\mathrm{T}}\right.$ ), las tasas de pérdidas $(-\Delta \mathrm{C})$ y/o ganancias de $\mathrm{C}$ del suelo $(\Delta \mathrm{C})$, las emisiones de gases de efecto invernadero (GEI) y remociones (-GEI) de $\mathrm{CO}_{2}$ atmosférico asociadas con algunas propiedades fisicoquímicas de los suelos en sistemas productivos de la Altillanura y otros de Piedemonte de la Orinoquia conformaron tres grupos bien definidos. El clúster I agrupó a pasturas mejoradas de Granada (S1) y sistemas agroforestales (SAFs) de café asociados con plátano y leguminosas de Villavicencio (S9), donde las ganancias de $\mathrm{C}(\Delta \mathrm{C})$ y absorciones de $\mathrm{CO}_{2}$ atmosférico (-GEI) fueron medias variando de $\approx 0.11$ a 2.37 t C ha $^{-1}$ año- $^{-1}$ y de -1.60 a -8.70 t CO 2 eq ha ${ }^{-1}$ año $^{-1}$; siendo que monocultivos en rotación también formaron parte de este grupo; el clúster II reunió a monocultivos de arroz de Villavicencio (S10) y de piña de Puerto López (S14) que presentaron las más altas pérdidas de C (- $\Delta \mathrm{C}$ ) del suelo y emisiones de $\mathrm{CO}_{2}$ atmosférico (GEI) de $\approx-2.08 \mathrm{a}-2.35$ t C ha- $\mathrm{año}^{-1}$ y de $\approx 7.62$ a $8.62 \mathrm{t} \mathrm{CO}_{2} \mathrm{eq} \mathrm{ha} \mathrm{haño}^{-1}$; el clúster III agrupo a sistemas agroforestales SAFs de caucho y leguminosas de cobertura (S13) y sistemas silvopastoriles (SSPs) de Acacia mangium y pasturas mejoradas (S12) de Puerto López con las más altas ganancias de $\mathrm{C}$ del suelo $(\Delta \mathrm{C})$ y absorciones de $\mathrm{CO}_{2}$ atmosférico $(-\mathrm{GEI})$ de $\approx 0.373$ a 2.64 t C ha ${ }^{-1}$ año $^{-1}$ y de $\approx-1.36$ a -9.67 t CO$_{2}$ eq ha ${ }^{-1}$ año ${ }^{-1}$. Los sistemas agroforestales son una buena alternativa para el secuestro de $\mathrm{C}$ del suelo en la Altillanura Plana de Colombia.
\end{abstract}

Palabras clave: Agricultura sostenible, cambio climático, entrada de residuos, tipo de labranza, usos del suelo

\begin{abstract}
The different soil management affects soil $\mathrm{C}$ stocks. Multivariate analysis of the modeling of soil $\mathrm{C}$ stocks (SOC0-T), soil $\mathrm{C}$ losses rates $(-\Delta \mathrm{C})$ and / or soil $\mathrm{C}$ gains $(\Delta \mathrm{C})$, greenhouse emissions $(\mathrm{GHG})$ and removals (-GHG) atmospheric of $\mathrm{CO}_{2}$ associated with some physicochemical properties of soils in productive systems of High plains and other of lower moun-
\end{abstract}


tains of the Orinoco region formed three distinct groups. Cluster I grouped improved pastures of Granada (S1) and coffee agroforestry systems (AFS) associated with banana and legumes of Villavicencio (S9), where gains of $\mathrm{C}(\Delta \mathrm{C})$ and removal of atmospheric $\mathrm{CO}_{2}$ (-GEI) were averages ranging from $\approx 0.11$ to $-2.37 \mathrm{t} \mathrm{C} \mathrm{ha}^{-1} \mathrm{yr}^{-1}$ and -1.60 to $-8.70 \mathrm{t} \mathrm{CO}_{2} \mathrm{eq} \mathrm{ha}^{-1} \mathrm{yr}^{-1}$; monocultures in rotation also were part of this group; Cluster II grouped rice monoculture of Villavicencio (S10) and pineapple of Puerto Lopez (S14) who had the highest losses of $\mathrm{C}(-\Delta \mathrm{C})$ and soil atmospheric $\mathrm{CO}_{2}$ emissions (GHG) of $\approx-2.08$ to -2.35 t C ha $\mathrm{yr}^{-1}$ of $\approx 7.62$ to 8.62 t CO $2 \mathrm{eq} \mathrm{ha-1} \mathrm{yr}^{-1}$; Cluster III grouped to agroforestry system AFS of rubber and leguminous cover crops (S13) and silvopastoral systems (SSPs) of Acacia mangium and improved pastures of Puerto Lopez (S12) with the highest gains of soil $\mathrm{C}(\Delta \mathrm{C})$ and removals of atmospheric $\mathrm{CO}_{2}$ (-GEI) of $\approx 0.373$ to 2.64 t $\mathrm{C} \mathrm{ha-1} \mathrm{yr}^{-1}$ and -1.36 to -9.67 $\approx \mathrm{t} \mathrm{CO}_{2} \mathrm{eq} \mathrm{ha} \mathrm{hr}^{-1} \mathrm{yr}^{-1}$. Agroforestry systems are a good alternative for soil C sequestration in Altillanura Plana of Colombia.

Keywords: Climate change, land use, residues inputs, sustainable agriculture, tillage type

\begin{abstract}
Resumo
A diferente gestão dos solos modifica os estoques do C orgânico do solo. A análise multivariada da modelagem do estoque de $\mathrm{C}$ orgânico $\left(\mathrm{SOC}_{0-\mathrm{T}}\right)$, as taxas de perdida $(-\Delta \mathrm{C})$ e / o aumento de $\mathrm{C}$ no solo $(\Delta \mathrm{C})$, as emissões de gases de efeito estufa (GEE) e remoções (-GEE) de $\mathrm{CO}_{2}$ atmosférico associado com algumas propriedades físico-químicas dos solos em sistemas produtivos da Altillanura e outras regiões de Piedemonte da região do Orinoco conformaram três grupos distintos. Cluster I agrupou pastagens melhoradas (S1) e sistemas agroflorestais (SAFs) de café associados com banana e leguminosas de Villavicencio $(S 9)$, onde o aumento de $\mathrm{C}(\Delta \mathrm{C})$ e remoção do $\mathrm{CO}_{2}$ atmosférico (-GEE) eram médias que variou entre $\approx 0,11$ até 2,37 t $\mathrm{C} \mathrm{ha}{ }^{-1}$ ano $^{-1}$ e -1,60 até -8,70 t $\mathrm{CO}_{2}$ eq ha ${ }^{-1}$ ano ${ }^{-1}$; as monoculturas em rotação também foram parte deste grupo; Cluster II agrupou a monocultura de arroz de Villavicencio (S10) e monocultura de abacaxi de Puerto Lopez (S14), que teve a maior perdida de $\mathrm{C}(-\Delta \mathrm{C})$ no solo e as emissões de $\mathrm{CO}_{2}$ na atmosfera $(\mathrm{GEE}) \approx-2,08$ até $-2,35$ t $\mathrm{C}$ ha $^{-1}$ ano ${ }^{-1}$ e $\approx$ 7,62 até 8,62 t CO 2 eq ha-1 ano $^{-1}$; Cluster III agrupou os sistemas agroflorestais SAFs de borracha e culturas de cobertura de leguminosas (S13), e sistemas silvipastoris (SSPs) de Acacia mangium e pastagens melhoradas (S12) de Puerto Lopez com o maior acumulo do $\mathrm{C}$ do solo $\mathrm{C}(\Delta \mathrm{C})$ e remoções de $\mathrm{CO}_{2}$ atmosférico (-GEE) de $\approx 0,373$ até 2,64 t $\mathrm{C}$ ha- ${ }^{-1}$ ano ${ }^{-1}$ e de $\approx-1,36$ até $-9,67$ t CO 2 eq ha $^{-1} \mathrm{ano}^{-1}$. Os sistemas agroflorestais são uma boa alternativa para o sequestro do $\mathrm{C}$ no solo na Altillanura Plana da Colômbia.
\end{abstract}

Palavras-Chave: Agricultura sustentável, entradas de resíduos, mudanças climáticas, tipo do preparo do solo, uso do solo

\section{Introducción}

La fijación de carbono orgánico en la materia orgánica del suelo (MOS) contribuye a la disminución de gases de efecto invernadero (GEI) provenientes de la agricultura (Lal, 2004), ocasionados por los incrementos de dióxido de carbono $\left(\mathrm{CO}_{2}\right)$ atmosférico, el mayor GEI causante del calentamiento global (IPCC, 2007).

A nivel global, se estima que la agricultura contribuye aproximadamente con el $22 \%$ de las emisiones totales de $\mathrm{CO}_{2}$ (IPCC, 2007). De los gases emitidos a la atmósfera, el $\mathrm{CO}_{2}$ es el que presenta la mayor contribución relativa. En 2007, de las emisiones totales de $\mathrm{GEI}$, el $\mathrm{CO}_{2}$ representó un $77 \%$ con respecto a un 14 y $8 \%$ del metano y óxido nitroso (en términos de $\mathrm{CO}_{2}$ equivalente), debido principalmente a cambios en los usos del suelo.

Dentro del macro proyecto de investigación "Plan de manejo de la fertilidad de los suelos de la Altillanura" adscrito a la Dirección de Investigaciones de la Universidad de los Llanos, se contempló el estudio de "Modelación de los stocks de $\mathrm{C}$ y las emisiones de dióxido de $C$ en sistemas productivos de la Altillanura Plana, Meta, Colombia".

La Altillanura Plana con pendientes inferiores a $1 \%$ ocupa más del $90 \%$ del área de la Orinoquia y se caracteriza por una vegetación de sabana; se extiende al sur del río Meta desde el municipio de Puerto López hasta el límite con Venezuela, formada principalmente por sedimentos aluviales del Pleistoceno primario (Cochrane et al., 1985).

Dependiendo de los factores de manejo de los suelos a que están sometidos los diversos sistemas de producción, éstos pueden actuar como sumideros o fuentes de gases de efecto invernadero (GEI) (IPCC, 2006; Lal, 2010), según la forma en la que las prácticas específicas influyan sobre las entradas y salidas de $\mathrm{C}$ de los suelos en los sistemas (Dossa et al., 2008; Andrade et al., 2013; Somarriba et al., 2013).

En la Altillanura Plana del Meta, y en general en todas las áreas de los Llanos Orientales, se viene desarrollando una producción agrícola y pecuaria altamente intensificada, confirmándose así que más de 12 millones de hectáreas están sembradas en maíz, sorgo y soya 
como monocultivos (Vera, 2004), lo que ha generado la degradación de las propiedades físicas, químicas y biológicas de los suelos (Amézquita et al., 1997).

El cultivo de arroz es principalmente de secano, donde los departamentos del Meta y Casanare conjuntamente representan el $57.9 \%$ del total nacional (Benavides, 2010); su producción se da en forma mecanizada (con labranza convencional); en estas áreas aunque la labranza es una de las prácticas más realizada en el manejo de los suelos, ha tenido poca importancia sí se la compara con la que se le ha prestado a los problemas de acidez y de fertilidad en los suelos tropicales (Amézquita, 2013).

Por otra parte, la ganadería extensiva utiliza $87.38 \%$ del suelo disponible en el departamento del Meta (4.68 millones de hectáreas) (Benavides, 2010), principalmente ocupada en sabanas nativas y/o pasturas introducidas, la mayoría de ellas en avanzado estado de degradación (Amézquita et al., 2002).

Según Amézquita (2013), los suelos de la Altillanura Plana son frágiles y tienen alta susceptibilidad a problemas de compactación y erosión, principalmente en sistemas productivos ganaderos sometidos a sobrepastoreo, ya que la capa arable es muy reducida, siendo muy susceptibles a degradación, con efectos negativos en la sostenibilidad de estos sistemas.

La Orinoquia tiene un área de 8.2 millones de hectáreas con aptitud forestal; el Meta en el 2007 contribuyó solo con 4.000 hectáreas sembradas con árboles perennes principalmente para reforestación de tipo comercial (Benavides, 2010); con altas posibilidades para la implementación de sistemas agroforestales (Benavides, 2010).

En la modelación de los stocks de C relacionados con las emisiones de GEl, para estimar cambios en las existencias de $\mathrm{C}$ del suelo, se usan una serie de coeficientes o valores por defecto, asociados a factores del suelo (IPCC, 2006; Kaul et al., 2009) y las características biofísicas, como por ejemplo el clima o la zona ecológica (Ogle et al., 2005; IPCC, 2006).

Los diferentes usos del suelo (FLU) y las prácticas agrícolas que involucran factores de manejo del suelo, como tipos de labranza (FMG) y la disponibilidad de residuos $(\mathrm{FI})$, pueden ocasionar aumentos o disminuciones de los contenidos de $\mathrm{C}$ del suelo, dependientes de la tasa de mineralización de la materia orgánica del suelo (MOS) (IPCC, 2006; Six et al., 2006).

Los cambios de uso del suelo, tipos de labranza y entrada de residuos no solamente afectan la acumu- lación del carbono orgánico del suelo (IPCC, 2006; Reicosky and Archer, 2007; Smith et al., 2008), sino también otras propiedades fisicoquímicas de los suelos (La Scala et al., 2000; Liu et al., 2005), modificándose las emisiones de $\mathrm{CO}_{2}$ a la atmósfera (IPCC, 2006).

La agricultura conservacionista genera un equilibrio positivo entre las entradas y salidas de $\mathrm{C}$ del suelo en los sistemas (Lal, 2004; Cerri et al., 2007); objetivando así la preservación y aumento de los contenidos de la MOS, un mayor reciclaje de nutrientes, fijación biológica de nitrógeno, retención de agua y reducción en los procesos erosivos (Lovato et al., 2014).

La hipótesis formulada es que los diferentes manejos de los suelos en los sistemas productivos de la Altillanura Plana modifican los stocks de $\mathrm{C}$ del suelo y las emisiones de dióxido de carbono a la atmosfera, encontrándose sistemas productivos más sostenibles ambientalmente que otros.

El objetivo general de esta investigación fue hacer una modelación de los stock de $\mathrm{C}$ de los suelos y las emisiones de $\mathrm{CO}_{2}$ a la atmosfera asociada a algunas propiedades físicas de los suelos, influenciada por factores de manejo de los suelos en sistemas productivos de la Altillanura Plana y otros de piedemonte del departamento del Meta, Orinoquia, Colombia; con el fin de identificar los sistemas con un enfoque conservacionista de manejo del suelo que promuevan un aumento de la materia orgánica del suelo (MOS), que generen un balance positivo de $\mathrm{C}$ del suelo y negativo de las emisiones de $\mathrm{CO}_{2}$ a la atmósfera, contribuyendo en parte a mitigar los efectos del cambio climático global.

\section{Metodología}

El estudio se realizó en dos zonas de la Orinoquia colombiana: Piedemonte y Altillanura. En el primero se seleccionaron las localidades (Granada y Villavicencio) y en el segundo una localidad (Puerto López) (Tabla 1). En cada zona se seleccionaron cinco sistemas productivos representativos.

Por zona se incluyeron 20 fincas, que incluyeron uno o más de los sistemas seleccionados en cada una de las zonas; los suelos se muestrearon a $0.30 \mathrm{~m}$ de profundidad en 10 puntos y se extrajo una muestra compuesta para análisis de tipo fisicoquímicos como se muestra en la Tabla 1.

En las 20 fincas por zona se realizó una caracterización de los sistemas en cuanto a los factores de manejo de los suelos correspondientes a uso de la tierra (FLU), tipo de labranza (FMG) y las entradas de $C$ por residuos (FI) (Tabla 2), con el fin de unificar los valores por 
Tabla 1. Tipo de parámetros, metodologías y variables asociadas a las propiedades físico-químicas de los suelos muestreados y las emisiones de $\mathrm{CO}_{2}$ a la atmósfera en diferentes sistemas de la Altillanura Plana, Meta, Colombia, 2015.

\begin{tabular}{|c|c|c|c|c|}
\hline Parámetro & Unidad & Método & $\begin{array}{l}\text { Denominación } \\
\text { variable }\end{array}$ & Autor \\
\hline Densidad real & $\mathrm{g} / \mathrm{cc}$ & Picnómetro & $\mathrm{Dr}$ & IGAC (2006) \\
\hline Materia orgánica & $\%$ & Walkley Black & $\mathrm{MO}$ & IGAC (2006) \\
\hline Densidad aparente & g/cc & Terrón parafinado & $\mathrm{Da}$ & IGAC (2006) \\
\hline Porosidad & $\%$ & Relación de densidades & $\mathrm{Pt}$ & IGAC (2006) \\
\hline Microporosidad & $\%$ & $\begin{array}{l}\text { Platos de presión humedad } \\
\text { volumétrica a }-0.3 \text { bares }\end{array}$ & $\mathrm{H} \Theta \mathrm{cc}$ & IGAC (2006) \\
\hline Macroporosidad & $\%$ & $\begin{array}{l}\text { Por diferencia entre Porosidad } \\
\text { y microporosidad }\end{array}$ & M & IGAC (2006) \\
\hline Arena & $\%$ & Bouyoucos & A & Jaramillo (2006) \\
\hline Limo & $\%$ & Bouyoucos & L & Jaramillo (2006) \\
\hline Arcilla & $\%$ & Bouyoucos & $\mathrm{Ar}$ & Jaramillo (2006) \\
\hline $\begin{array}{l}\text { Coeficiente de } \\
\text { dispersión de arcillas }\end{array}$ & $\%$ & $\begin{array}{l}\text { Comparación entre el método de } \\
\text { bouyucos y el método sin dispersante }\end{array}$ & CD & Jaramillo (2006) \\
\hline C orgánico & $\%$ & MOS/1.726 & $\mathrm{CO}$ & IGAC (2006) \\
\hline Stock $C$ inicial & $\mathrm{tha}^{-1}$ & $\%$ CO total x Peso ha suelo $\times$ Da/10 & $\mathrm{COS}_{0}$ & IPCC (2006) \\
\hline Stock C final & $\mathrm{tha}^{-1}$ & $\left(\mathrm{SOC}_{0}\right) \times \mathrm{FLU} \times \mathrm{FMG} \times \mathrm{FI}$ & $\mathrm{COS}_{0-\mathrm{T}}$ & IPCC (2006) \\
\hline $\begin{array}{l}\text { Tasa de perdida y/o } \\
\text { ganancia de } \mathrm{C}\end{array}$ & 1 año $^{\text {tha }^{-}}$ & $\left(\mathrm{SOC}_{0}\right)-\mathrm{SOC}(0-\mathrm{T}) / 20-1$ & $-\Delta \mathrm{C}$ y/o $\Delta \mathrm{C}$ & IPCC (2006) \\
\hline $\begin{array}{l}\text { Emisiones y/o } \\
\text { absorciones de } \mathrm{CO} 2\end{array}$ & 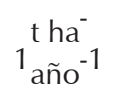 & $(\Delta \mathrm{C}) \times 3.67$ & GEl y -GEl & IPCC (2006) \\
\hline
\end{tabular}

defecto de pérdida y/o ganancia de C del suelo, sobre la base de la clasificación de la gestión del suelo sugeridas en el volumen 4 de las Directrices IPCC, capitulo 5 Tierras de Cultivo, cuadro 5.5 y capitulo 6 Pastizales, cuadro 6.2, con una proyección de 20 años bajo condiciones de zona tropical húmeda (IPCC, 2006).

Las existencias de $\mathrm{C}$ del suelo de referencia $\left(\mathrm{SOC}_{0}\right)$ se multiplicaron por los valores por defecto (FLU, FMG, FI) considerados para estimar las existencias «finales» de C orgánico del suelo (SOC (O-T)) que representan las condiciones en el último año de inventario (año 20) (IPCC, 2006),

Las tasas de ganancia $(\Delta C)$ y/o pérdida de $C$ del suelo $(-\Delta \mathrm{C})$ para los diferentes sistemas productivos de piedemonte y la Altillanura, se estimaron como los cambios anuales promedio de existencias de $C$ orgánico del suelo restando las existencias «iniciales» de C orgánico del suelo (SOC$)$, de las existencias «finales» de $\mathrm{C}$ orgánico del suelo $\left(\mathrm{SOC}_{0-\mathrm{T}}\right)$ dividido por la de- pendencia en el tiempo de los factores de cambios de existencias (es decir, 20 años empleando los factores por defecto) (IPCC, 2006).

Por último, para transformar la tasa de pérdidas y/o ganancias de $\mathrm{C}$ a emisiones y/o absorciones de dióxido de carbono en $\mathrm{kgCO}_{2}$ eq (GEI) ésta se multiplicó por el factor 3.67 (IPCC, 2006).

\section{Análisis estadístico}

El análisis estadístico se realizó mediante un análisis multivariado utilizando la técnicas de ACP para reducir la dimensionalidad del problema y la de agrupamiento jerárquico utilizando como criterio las distancias de Ward (Manly, 1997).

\section{Resultados y discusión}

La tabla 3 presenta la modelación de los stocks de C proyectados a 20 años $\left(\mathrm{SOC}_{0-\mathrm{T}}\right)$, las tasas de pérdidas 
Tabla 2. Caracterización de los sistemas en cuanto a uso del suelo (FLU), tipo de labranza (FMG), entradas por residuos (FI) en diferentes sistemas productivos de la Altillanura Plana, Meta, Colombia, 2015.

\begin{tabular}{|c|c|c|c|c|}
\hline \multirow[b]{2}{*}{ Zonas } & \multirow[b]{2}{*}{ Sistemas productivos } & \multicolumn{3}{|c|}{ Niveles asociados a los factores de manejo de suelo } \\
\hline & & (Uso del suelo) (FLU) & $\begin{array}{l}\text { (Laboreo) } \\
\text { (FMG) }\end{array}$ & $\begin{array}{l}\text { (Entrada de } \\
\text { residuos) } \\
(\mathrm{FI})\end{array}$ \\
\hline \multirow{5}{*}{ Granada } & $\mathrm{S} 1$ = Pastura mejorada & $\begin{array}{l}\text { Pastizal gestionado } \\
\text { sosteniblemente }^{1}\end{array}$ & Reducido $^{6}$ & Media $^{9}$ \\
\hline & $\begin{array}{l}\mathrm{S} 2 \text { = Monocultivo de soya } \\
\text { en rotación con maíz }\end{array}$ & $\begin{array}{l}\text { Cultivos de largo plazo } \\
\text { gestionado sosteniblemente }\end{array}$ & Reducido $^{6}$ & Media $^{9}$ \\
\hline & $\begin{array}{l}\text { S3 = Cultivo de maíz en } \\
\text { rotación con yuca }\end{array}$ & $\begin{array}{l}\text { Cultivos de largo plazo } \\
\text { gestionado sosteniblemente }^{2}\end{array}$ & Reducido $^{6}$ & Media $^{9}$ \\
\hline & $\begin{array}{l}\text { S4 = Monocultivo de plátano } \\
\text { en rotación con yuca }\end{array}$ & $\begin{array}{l}\text { Cultivos de largo plazo } \\
\text { gestionado sosteniblemente }\end{array}$ & Reducido $^{6}$ & Media $^{9}$ \\
\hline & $\begin{array}{l}\text { S5 = Monocultivo de } \\
\text { arroz en rotación con } \\
\text { soya y maíz }\end{array}$ & $\begin{array}{l}\text { Cultivos de largo plazo } \\
\text { gestionado sosteniblemente }\end{array}$ & Reducido $^{6}$ & Media $^{9}$ \\
\hline \multirow{5}{*}{ Villavicencio } & $\mathrm{S} 6$ = Pastura mejorada & $\begin{array}{l}\text { Pastizal gestionado } \\
\text { sosteniblemente }^{1}\end{array}$ & Reducido $^{6}$ & Media $^{9}$ \\
\hline & $\begin{array}{l}\text { S7 = Sistema agroflorestal de } \\
\text { cacao y Acacia mangium }\end{array}$ & $\begin{array}{l}\text { Cultivo de perenne gestionado } \\
\text { sosteniblemente }^{3}\end{array}$ & Sin laboreo ${ }^{5}$ & Alta $^{10}$ \\
\hline & S8 = Monocultivo de piña & $\begin{array}{l}\text { Cultivo de largo plazo } \\
\text { gestionado continuamente }\end{array}$ & Total $^{7}$ & Baja $^{11}$ \\
\hline & $\begin{array}{l}\text { S9 = Sistema agroforestal de } \\
\text { café con plátano y leguminosas }\end{array}$ & $\begin{array}{l}\text { Cultivo de perennes gestionado } \\
\text { sosteniblemente }^{3}\end{array}$ & Reducido $^{6}$ & Alta $^{10}$ \\
\hline & S10 = Monocultivo de arroz & $\begin{array}{l}\text { Cultivos de largo plazo } \\
\text { gestionado continuamente }\end{array}$ & Total $^{7}$ & Baja $^{11}$ \\
\hline \multirow{5}{*}{ Pto. Lopez } & S11 = Bosque secundário & De reserva $(<20 \text { años })^{5}$ & Sin laboreo $^{5}$ & Alta $^{8}$ \\
\hline & $\begin{array}{l}\text { S12 = Sistema silvo pastoril } \\
\text { de Acacia mangium y } \\
\text { pastura mejorada }\end{array}$ & $\begin{array}{l}\text { Pastizal gestionado } \\
\text { sosteniblemente }^{1}\end{array}$ & Reducido $^{8}$ & Alta ${ }^{8}$ \\
\hline & $\begin{array}{l}\text { S13 = Sistema agroforestal } \\
\text { de caucho y leguminosas } \\
\text { de cobertura }\end{array}$ & $\begin{array}{l}\text { Cultivo de perennes gestionado } \\
\text { sosteniblemente }^{3}\end{array}$ & Reducido $^{8}$ & Alta ${ }^{8}$ \\
\hline & S14 = Monocultivo de piña & $\begin{array}{l}\text { Cultivos de largo plazo } \\
\text { gestionado continuamente }\end{array}$ & Total $^{9}$ & Baja $^{11}$ \\
\hline & S15 = Monocultivo de cacao & $\begin{array}{l}\text { Cultivo de perennes gestionado } \\
\text { sosteniblemente }^{3}\end{array}$ & Reducido $^{8}$ & Media $^{9}$ \\
\hline
\end{tabular}

$1 \quad$ (FLU1)=Pastizales con pastoreo moderado y que recibe al menos una mejora (p. ej., fertilización, mejora de especies, inclusión de árboles ${ }^{2}$ (FLU2) $=$ El factor de uso de la tierra se estimó con relación a laboreo reducido y a niveles nominales («medios») de entrada de carbono ${ }^{3}$ (FLU5) $=$ Cultivos de árboles perennes a largo plazo que incluyen alguna mejora; ${ }^{4}($ FLU4) $=$ Representa la superficie gestionada en forma continua durante más de 20 años, predominantemente con cultivos anuales ${ }^{5}($ FMG 1$)=\operatorname{Sin}$ laboreo; ${ }^{6}($ FMG2 $)=$ Laboreo reducido; ${ }^{7}($ FMG2 $)=$ Laboreo Total; ${ }^{8}(\mathrm{FI} 1)=$ alta entrada de residuos; ${ }^{9}(\mathrm{FI} 2)=$ media entrada de residuos; ${ }^{10}(\mathrm{Fl} 3)$ = baja entrada de residuos. 
Tabla 3. Modelación de los stocks de $\mathrm{C}$ finales $\left(\mathrm{SOC}_{0} \mathrm{-T}\right)$ y emisiones y/o absorciones de dióxido de carbono a la atmósfera (GEI) considerando los factores uso de suelo (FLU), tipo de labranza (FMG) y entrada de residuos (FI) asociados a los sistemas productivos de la Altillanura Plana del Meta, Colombia, 2015.

\begin{tabular}{|c|c|c|c|}
\hline Sistemas & $\begin{array}{c}\text { Tasas de ganancias }(\Delta C) \\
\text { y/o pérdidas de } C(-\Delta C) \\
\text { t } \mathbf{C} \text { ha }^{-1} \text { año } \\
\end{array}$ & $\begin{array}{c}\text { Stock de C final }\left(\mathrm{SOC}_{0-\mathrm{T}}\right) \\
\text { en } 20 \text { años } \\
\text { t C ha-1 }\end{array}$ & $\begin{array}{c}\text { Emisiones de } \mathrm{CO}_{2} \text { (GEI) y/o } \\
\text { absorciones (-GEI) } \\
\text { t } \mathrm{CO}_{2} \text { eqha }^{-1} \text { año }^{-1}\end{array}$ \\
\hline S1 & 0.11 & 75.72 & -1.60 \\
\hline $\mathrm{S} 2$ & 0.34 & 66.15 & -1.26 \\
\hline S3 & 0.25 & 49.48 & -0.94 \\
\hline S4 & 0.29 & 53.55 & -1.02 \\
\hline S5 & -0.14 & 43.63 & 0.53 \\
\hline S6 & 0.38 & 74.49 & -1.42 \\
\hline S7 & 0.39 & 75.45 & -1.44 \\
\hline S8 & -2.22 & 34.80 & 8.15 \\
\hline S9 & 2.37 & 104.90 & -8.70 \\
\hline S10 & -2.08 & 31.32 & 7.62 \\
\hline S11 & 0.42 & 81.96 & -1.57 \\
\hline S12 & 2.64 & 116.64 & -9.67 \\
\hline S13 & 0.37 & 71.61 & -1.36 \\
\hline S14 & -2.35 & 36.82 & 8.62 \\
\hline S15 & 0.18 & 57.73 & -0.66 \\
\hline
\end{tabular}

S1 = Pastura mejorada; S2 = Monocultivo de soya en rotación con maíz; S3 = Cultivo de maíz en rotación con yuca; S4= Monocultivo de plátano en rotación con yuca; S5= Monocultivo de arroz en rotación con soya y maíz; S6= Pastura mejorada; S7= Sistema agroforestal de cacao con Acacia mangium; S8= Monocultivo de piña; S9= Sistema agroforestal de café asociado con plátano y leguminosas; S10= Monocultivo de arroz; S11= Bosque secundario; S12= Sistema silvopastoril de Acacia mangium y pastura mejorada; S13 = Sistema agroforestal de caucho y leguminosas de cobertura; S14 = Monocultivo de piña; S15 = Monocultivo de cacao.

de $C(-\Delta C)$ y ganancias de $C(\Delta C)$ del suelo y las emisiones (GEI) y/o sumideros de $\mathrm{CO}_{2}$ (-GEI) a la atmósfera en los diferentes sistemas caracterizados en zonas de piedemonte y Altillanura.

La tabla 4 presenta una descripción estadística de las variables seleccionadas en el análisis multivariado relacionadas con las propiedades fisicoquímicas del suelo asociadas a esas emisiones y/o absorciones de $\mathrm{CO}_{2}$ a la atmósfera.

En las características fisicoquímicas del suelo asociadas a las emisiones y/o absorciones de GEI en los sistemas de Piedemonte y Altillanura de la Orinoquia, se puede afirmar que existió una baja variabilidad en la materia orgánica del suelo (MOS) considerada entre baja a alta (1.60-4.20\%); mientras la distribución de

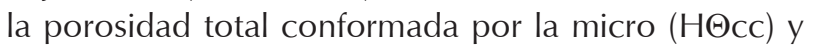
macro (M) porosidad varió entre $7.35-38.22 \%$ y 9.30 $42.63 \%$ respectivamente; Sánchez y Salinas (1983) han encontrado que muchos Oxisoles y Ultisoles tropicales tienen una baja capacidad de almacenamiento de agua $(\mathrm{H \Theta cc})$, debido al tipo de coloide inorgánico que domina en ellos y al bajo contenido de materia orgánica; los bajos valores de macroporosidad ( $M$ < 10\%) (Jaramillo, 2002; Basamba et al., 2006) estarían confirmando problemas de compactación en algunos de los sistemas evaluados. La incorporación de leguminosas en una pastura nativa de los Llanos Orientales mejoró la porosidad total de 29.6 a $30.8 \%$ en los primeros $30 \mathrm{~cm}$ de suelo (Amézquita et al., 2002).

Los contenidos de Arena (A), limo (L) y arcilla (Ar) variaron entre 9-40, 10-55 y $14-72 \%$, respectivamente, indicando una alta variabilidad en la composición mineralógica de estos suelos, correspondiendo principalmente a Oxisoles y Ultisoles (Malagon, 2003). El valor máximo para coeficiente de dispersión (CD) fue de $35.12 \%$, indicando susceptibilidad a procesos erosivos (CD >25\%) (Jaramillo, 2002) (Tabla 4). La cantidad relativa de arcilla que se encuentre dispersa, es decir, sin unirse a otras partículas del suelo, da una idea del grado de agregación y de la estabilidad de los agregados que tiene el suelo (Castillo et al., 2000). Los contenidos de carbono orgánico (CO) presentaron niveles de bajos a medios entre $0.92-2.90 \%$, valores que influyeron en los stocks de $\mathrm{C}$ iniciales $\left(\mathrm{COS}_{0}\right)$ que variaron entre 44.58-81.61 t C ha-1 (Tabla 4). 
Tabla 4. Variables seleccionadas en el análisis multivariado correspondientes a las propiedades fisicoquímicas de los suelos y las emisiones de GEI de los diferentes sistemas productivos de la Altillanura Plana, Meta, Colombia, 2015.

\begin{tabular}{|c|c|c|c|c|c|}
\hline $\begin{array}{l}\text { Tipo de variable } \\
\text { seleccionada }\end{array}$ & Unidad & Media & $\begin{array}{l}\text { Desviación } \\
\text { estándar }(\delta)\end{array}$ & Mínimo & Máximo \\
\hline MOS & $\%$ & 2.88 & 0.73 & 1.60 & 4.20 \\
\hline 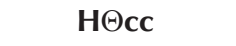 & $\%$ & 25.54 & 8,00 & 7.35 & 38.22 \\
\hline $\mathbf{M}$ & $\%$ & 25.42 & 6.97 & 9.30 & 42.63 \\
\hline A & $\%$ & 25.80 & 10.88 & 9.00 & 40.00 \\
\hline $\mathbf{L}$ & $\%$ & 31.53 & 14.49 & 10.00 & 55.00 \\
\hline Ar & $\%$ & 42.40 & 22.87 & 14.00 & 72.00 \\
\hline CD & $\%$ & 12.99 & 9.82 & 1.70 & 35.12 \\
\hline $\mathrm{CO}$ & $\%$ & 1.72 & 0.51 & 0.92 & 2.90 \\
\hline $\mathrm{COS}_{0}$ & t C ha-1 & 64.10 & 10.98 & 44.58 & 81.61 \\
\hline $\operatorname{COS}_{0-\mathrm{T}}$ & t C ha-1 & 64.57 & 24.01 & 31.32 & 116.68 \\
\hline$\Delta \mathbf{C}$ & t C ha-1 año $^{-1}$ & 0.03 & 1.37 & -2.35 & 2.64 \\
\hline GEI & $\mathrm{tCO}_{2}$ eq ha $\mathrm{ha}^{-1} \mathrm{año}^{-1}$ & -2.39 & 10.54 & -9.67 & 8.62 \\
\hline
\end{tabular}

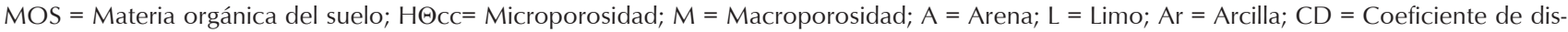
persión de arcillas; $\mathrm{CO}=$ Carbono orgánico; $\mathrm{SOC}_{0}=$ Stock inicial de $\mathrm{C} ; \mathrm{SOC}(\mathrm{O}-\mathrm{T})=$ Stock final; $\Delta \mathrm{C}=$ Tasa de perdida y/o ganancia de $\mathrm{C}$; $\mathrm{GEI}=$ Emisiones de $\mathrm{CO}_{2}$ a la atmósfera. $\Delta$

Desde el punto de vista de las características fisicoquímicas de los suelos, los suelos de la Orinoquia son susceptibles a problemas de sellamiento superficial, presentando baja infiltración y baja capacidad de almacenamiento de agua y alta escorrentía; propiedades asociadas principalmente a bajos contenidos de MOS (Amézquita et al., 2002) y alto coeficiente de dispersión de arcillas (Jaramillo, 2002).

Los factores de manejo de los suelos influyeron en una alta variabilidad de los stocks de $\mathrm{C}$ finales ( $\left.\mathrm{COS}_{0-\mathrm{T}}\right)$ con rangos entre 31.32-116.68 t C ha- en monocultivo de arroz de Villavicencio (S10) y sistema silvopastoril de pastura mejorada y Acacia mangium de Puerto López (S12), influyendo en rangos amplios de las tasas de pérdidas $(-\Delta C)$ y/o ganancias de $C$ del suelo $(\Delta C)$ entre -2.35 a $2.64 \mathrm{t} \mathrm{ha}^{-1}$ año $^{-1}$ respectivamente (Tabla 3 ). Conant et al., (2001) en cerca de 115 estudios en 17 países también reportó rangos amplios que variaron de $-0.2 \mathrm{a}+3.0 \mathrm{t} \mathrm{C} \mathrm{ha-}^{-1}$ año $^{-1}$ en sistemas de pasturas degradadas comparadas con recuperadas.

A diferencia de las reportadas por Amado et al., (2006), que fueron más estrechas, los autores indicaron que la tasa de secuestro de $\mathrm{C}$ varió de 0.12 a $1.6 \mathrm{t} \mathrm{C} \mathrm{ha}^{-1}$ año ${ }^{-1}$ comparando la labranza convencional con la labranza mínima. Lal et al., (1999), en suelos de Estados Unidos estimaron tasas de acumulación en el suelo que varían entre 0.10 a 0.50 t C ha ${ }^{-1}$ año $^{-1}$.

El rango más alto obtenido en los sistemas silvopastoriles (SPSs) de pasturas mejoradas con Acacia mangium de Puerto López (S12) fue superior a las reportadas por Bernoux et al., (2006) en monocultivos en rotación en una zona tropical; indicando que los SPSs son más eficientes en el secuestro de $\mathrm{C}$ del suelo.

Los amplios rangos encontrados relacionados con las tasas de ganancias $(\Delta C)$ y/o pérdidas de $C$ del suelo $(-\Delta C)$ influyeron en una variabilidad media $(\delta=10.54)$ (Tabla 4), en cuanto a las emisiones de $\mathrm{CO}_{2}$ a la atmósfera (GEI), con rangos entre 8.60 t C ha- ${ }^{-1}$ año $^{-1}$ y -9.67 t C ha ${ }^{-1}$ año $^{-1}$ en sistema silvopastoril (SPS) de pastura mejorada con Acacia mangium (S12) y monocultivo de piña (S14) de Puerto López (Tabla 4), correspondiendo a sistemas que actúan como sumideros y otros como emisores de $\mathrm{CO}_{2}$ a la atmósfera respectivamente.

En este sentido, los factores del suelo en sistemas agroforestales que aumentan las entradas de $\mathrm{C}$ y/o favorecen el almacenamiento de C (Kirby y Potvin, 2007; Nair et al., 2010), disminuyen las emisiones de $\mathrm{CO}_{2}$ atmosférico (Yoon et al., 2008).

\section{Matriz de correlaciones}

La matriz de correlaciones del análisis multivariado permitió analizar la relación entre las distintas variables fisicoquímicas del suelo y las emisiones de GEI (Tabla 5).

La modelación de las emisiones (GEI) y/o absorciones (-GEI) de $\mathrm{CO}_{2}$ atmosférico estuvieron asociadas inversamente con los stock de carbono finales (SOC $\left.\mathrm{SO}_{0-\mathrm{T}}\right)(\mathrm{r}=-$ 
$0.50)$ y las tasas de pérdidas $(-\Delta C)$ y/o ganancias de $C$ $(\Delta \mathrm{C})(\mathrm{r}=-0.54)$, debido a que generan respectivamente emisiones y/o absorciones de GEl a la atmósfera; a su vez las tasas de pérdidas $(-\Delta \mathrm{C})$ y/o ganancias de $C(\Delta \mathrm{C})$ y stock de $\mathrm{C}$ iniciales $\left(\mathrm{SOC}_{0}\right)$ con el coeficiente de dispersión de arcillas ( $r=-0.53$; $r=-0.52)$; a su vez éste con microporosidad $(\mathrm{m})$ y arcilla $(\mathrm{r}=-0.72 ; \mathrm{r}=-0,48)$; arcilla $(\mathrm{Ar})$ y limo (L) con arena (A) $(\mathrm{r}=-0,87 ;-0.92)$; $\operatorname{arena}(\mathrm{A})$

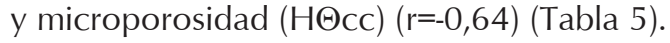

La mineralización de la materia orgánica es menor en suelos arcillosos que en suelos arenosos sometidos a labranza convencional y puede existir una alta estabilidad física de la materia orgánica asociada con minerales de arcilla en suelos altamente intemperizados (Bayer et al., 2006); en consecuencia, los Oxisoles de la Orinoquia estarían menos expuestos a procesos erosivos

Por otra parte, se encontró una relación proporcional directa entre las tasas de perdida y/o ganancia de $\mathrm{C}(-\Delta \mathrm{C}$ y/o $\Delta \mathrm{C})$ y los stocks de $\mathrm{C}$ finales $\left(\mathrm{SOC}_{0-\mathrm{T}}\right)(\mathrm{r}=$ 0.91); stocks de $\mathrm{C}$ iniciales (SOCo) con materia orgánica del suelo (MOS) y carbono orgánico (CO) $(r=0.70 ; r$ $=0.61)$; carbono orgánico (CO) y macroporosidad (M) $(\mathrm{r}=0.70) ; \operatorname{arcilla}(\mathrm{Ar})$ y limo (L) con microporosidad (m)

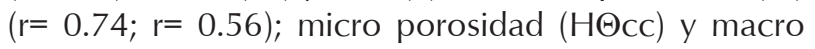
porosidad (M) con materia orgánica del suelo (MOS) $(r=0.52 ; r=0.56)$ (Tabla 5).

Esas relaciones pueden ser atribuidas a procesos que controlan la acumulación y preservación de la MOS y que varían principalmente dependiendo del tipo de suelo y su manejo, clima y sistemas implementados; es reconocido que la MOS es uno de los principales indicadores de calidad de suelo por estar relacionada positivamente con diferentes propiedades fisicoquímicas del suelo (Reeves, 1997).

Según Brady (2002) existe una alta heterogeneidad de los procesos fisicoquímicos de los suelos que, asociados a los factores ambientales, serían controladores de las emisiones de $\mathrm{CO}_{2}$ atmosférico; éstos resultan muy variables con coeficientes de variación reportados entre 100 a 300\% (Boeckx et al., 2005).

\section{Análisis de componentes principales (ACP)}

El ACP permitió identificar que los tres primeros factores principales fueron suficientes para explicar un $78.12 \%$ de la variabilidad total de los sistemas evaluados. La conformación de los tres factores está dada por la correlación de cada una de las variables, relacionadas con las propiedades fisicoquímicas del suelo y las emisiones de $\mathrm{CO}_{2}$, con el componente principal correspondiente (Tabla 6).

Las emisiones de $\mathrm{CO}_{2}$ a la atmósfera (GEI), el coeficiente de dispersión de arcillas (CD) y la fracción arena (A) contribuyeron de manera negativa en la conformación del CP1, y de manera positiva con las demás variables fisicoquímicas del suelo excepto la macro porosidad (M) y los stocks de $\mathrm{C}$ iniciales $\left(\mathrm{COS}_{0}\right)$. Así se explica el $38.39 \%$ de las variaciones en los sistemas (Tabla 6).

Los stocks de carbono iniciales ( $\left.\mathrm{COS}_{0}\right)$, el carbono orgánico del suelo (CO), la materia orgánica del suelo (MOS) y la macroporosidad (M) son las variables más

Tabla 5. Matriz de correlaciones de las variables analizadas en sistemas productivos de la Altillanura, Meta, Colombia, 2015.

\begin{tabular}{|c|c|c|c|c|c|c|c|c|c|c|c|c|}
\hline & MOS & 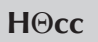 & $\mathbf{M}$ & A & L & Ar & CD & $\mathrm{CO}$ & $\operatorname{COS}_{0}$ & $\cos _{0-\mathrm{T}}$ & $\Delta C$ & GEI \\
\hline MOS & 1 & & & & & & & & & & & \\
\hline $\mathrm{H} \Theta \mathrm{CC}$ & 0.52 & 1 & & & & & & & & & & \\
\hline M & 0.56 & 0.15 & 1 & & & & & & & & & \\
\hline A & -0.34 & -0.64 & 0.03 & 1 & & & & & & & & \\
\hline L & 0.36 & 0.56 & 0.17 & -0.92 & 1 & & & & & & & \\
\hline $\mathrm{Ar}$ & 0.28 & 0.74 & -0.31 & -0.87 & 0.67 & 1 & & & & & & \\
\hline CD & 0.32 & -0.72 & -0.04 & 0.28 & -0.19 & -0.48 & 1 & & & & & \\
\hline $\mathrm{CO}$ & 0.94 & 0.46 & 0.70 & -0.26 & 0.34 & 0.16 & -0.35 & 1 & & & & \\
\hline $\mathrm{COS}_{0}$ & 0.70 & -0.14 & 0.23 & 0.11 & -0.14 & -0.09 & 0.12 & 0.61 & 1 & & & \\
\hline $\mathrm{COS}_{0-\mathrm{T}}$ & 0.30 & 0.34 & 0.03 & -0.07 & 0.10 & 0.14 & -0.52 & 0.29 & 0.02 & 1 & & \\
\hline$\Delta \mathrm{C}$ & -0.02 & 0.37 & -0.07 & -0.11 & 0.15 & 0.17 & -0.53 & 0.01 & -0.40 & 0.91 & 1 & \\
\hline GEI & -0.21 & -0.37 & -0.09 & 0.29 & -0.44 & -0.28 & 0.18 & -0.16 & 0.19 & -0.50 & -0.54 & 1 \\
\hline
\end{tabular}

MOS = Materia orgánica del suelo; $\mathrm{H \Theta cc}=$ Microporosidad; $\mathrm{M}=$ Macroporosidad; $\mathrm{A}=$ Arena; $\mathrm{L}=\mathrm{Limo} ; \mathrm{Ar}=$ Arcilla; $\mathrm{CD}=\mathrm{Coeficiente} \mathrm{de} \mathrm{dispersión}$ de arcillas; $\mathrm{CO}=$ Carbono orgánico; $\mathrm{SOC}_{0}=$ Stock inicial de $\mathrm{C} ; \mathrm{SOC}(\mathrm{O}-\mathrm{T})=$ Stock final; $\Delta \mathrm{C}=$ Tasa de perdida $\mathrm{y} / \mathrm{o}$ ganancia de $\mathrm{C} ; \mathrm{GEI}=\mathrm{Emisiones}$ de $\mathrm{CO}_{2}$ a la atmósfera. 
explicativas del CP2 en sentido positivo, explicando el $22.94 \%$ de las variaciones en los sistemas (Tabla 6).

En el análisis del tercer componente, la variables que más contribuyeron en sentido negativo con el mismo fueron los stocks de $\mathrm{C}$ finales $\left(\mathrm{COS}_{0-\mathrm{T}}\right)$, seguido de las tasas de pérdidas $(-\Delta \mathrm{C})$, y/o ganancias de $C(\Delta \mathrm{C})$, explicando así el $16.89 \%$ de las variaciones (Tabla 6 ), posiblemente permitiendo distinguir sistemas con estas dos características, especialmente el monocultivo de piña de Puerto López (S14).

Esas correlaciones se muestran en la Fig. 1, y su proyección en el gráfico están representadas por una flecha para cada propiedad fisicoquímica del suelo y las emisiones de GEl a la atmósfera.

\section{Análisis de clúster}

Los índices de similaridad entre las variables analizadas calculados con base en las distancias de Ward, permitieron agrupar los sistemas productivos analizados como se muestran en la Figura 2.

En el clúster I hay tres variables que caracterizaron mayormente a este grupo (MOS, CO y Stock $C_{0}$ ), agrupando a todos aquellos sistemas con tasas de secuestro de $\mathrm{C}(\Delta \mathrm{C})$ y absorciones de $\mathrm{CO}_{2}$ atmosférico (-GEI) medias que están recibiendo algún tipo de mejora y están siendo manejados sosteniblemente con laboreo reducido y entradas medias de residuos actuando como sumideros de C; los sistemas de pasturas mejoradas de Granada (S1) y de Villavicencio (S6) fueron similares entre sí a los sistemas agroforestales (SAFs) de café asociados con plátano y árboles de leguminosas encontrados en Villavicencio (S9) (Figura 2).

El secuestro de $\mathrm{C}$ por parte de los sistemas agroforestales SAFs implica la absorción de $\mathrm{CO}_{2}$ atmosférico durante la fotosíntesis y la transferencia del $\mathrm{C}$ fijado en la biomasa de árboles hacia el suelo, que garantiza un almacenamiento de C a largo plazo (Nair et al., 2010).

Ávila et al., (2001), encontraron que más del 89\% del C almacenado en sistemas agroforestales de café y pastos correspondió al $\mathrm{C}$ del suelo; éste varió entre el $90 \%$ (121 t C ha $\left.{ }^{-1}\right)$ en un sistema agroforestal de caféeucalipto de ocho años a 99.9\% (84 t C ha-1) en una pastura natural.

A su vez estos sistemas guardaron similitud con los monocultivos de plátano en rotación con yuca de Granada (S4), monocultivos de cacao de Puerto López (S15) y monocultivos de soya en rotación con maíz de Granada (S2) coincidiendo con los monocultivos de arroz en rotación con maíz y soya (S5) y monocultivos de maíz en rotación con yuca (S3) de Granada (Figura 2).

Tabla 6. Correlación entre los stocks de $C$ y emisiones de GEl asociadas a propiedades fisicoquímicas y los tres primeros componentes principales, en sistemas productivos de la Altillanura Plana, Meta, Colombia, 2015.

\begin{tabular}{|c|c|c|c|}
\hline Variables & CP1 & CP2 & CP3 \\
\hline MOS & 0.67 & 0.71 & -0.06 \\
\hline $\mathrm{H \Theta cc}$ & 0.87 & -0.11 & 0.09 \\
\hline M & 0.25 & 0.67 & -0.21 \\
\hline A & -0.75 & 0.17 & -0.59 \\
\hline L & 0.74 & -0.10 & 0.48 \\
\hline $\mathrm{Ar}$ & 0.75 & -0.31 & 0.49 \\
\hline$C D$ & -0.67 & 0.18 & 0.28 \\
\hline $\mathrm{CO}$ & 0.64 & 0.74 & -0.14 \\
\hline $\mathrm{COS}_{0}$ & 0.05 & 0.83 & -0.03 \\
\hline $\cos _{0-\mathrm{T}}$ & 0.55 & -0.20 & -0.73 \\
\hline$\Delta \mathrm{C}$ & 0.48 & -0.53 & -0.66 \\
\hline GEI & -0.55 & 0.26 & 0.26 \\
\hline
\end{tabular}

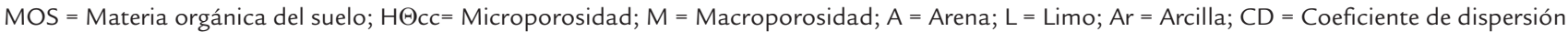
de arcillas; $\mathrm{CO}=$ Carbono orgánico; $\mathrm{SOC}_{0}=$ Stock inicial de $\mathrm{C} ; \mathrm{SOC}(0-\mathrm{T})=$ Stock final; $\triangle \mathrm{C}=$ Tasa de perdida $y /$ o ganancia de $C ; \mathrm{GEI}=\mathrm{Emisiones}$ de $\mathrm{CO}_{2}$ a la atmósfera. 


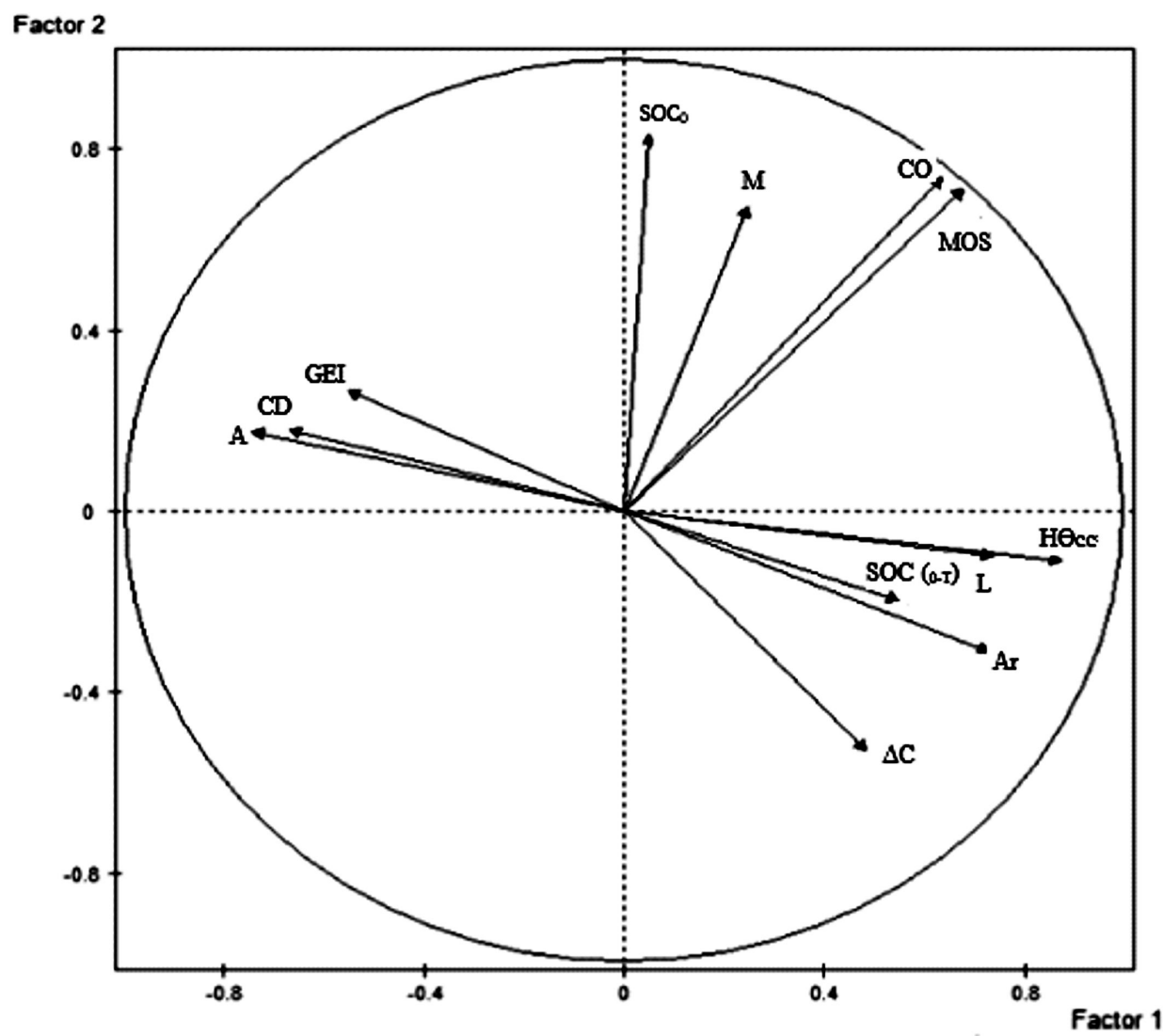

Figura 1. Diagrama de la relación entre variables analizadas en sistemas productivos de la Altillanura, Meta, Colombia, 2015. MOS = Materia orgánica del suelo; $\mathrm{H \Theta cc}=$ Micro porosidad; $\mathrm{M}=$ Macro porosidad; $\mathrm{A}=$ Arena; $\mathrm{L}=\mathrm{Limo} ; \mathrm{Ar}=\mathrm{Arcilla} ; \mathrm{CD}=\mathrm{Coefi}-$ ciente de dispersión de arcillas; $\mathrm{CO}=$ Carbono orgánico; $\mathrm{SOC}_{0}=$ Stock inicial de $\mathrm{C} ; \mathrm{SOC}(0-\mathrm{T})=\mathrm{Stock}$ final; $\triangle \mathrm{C}=\mathrm{Tasa}$ de perdida y/o ganancia de C; GEI = Emisiones de $\mathrm{CO}_{2}$ a la atmósfera.

En un cultivo de soya donde se realizó siembra directa y rotación con maíz con un alto aporte de residuos al suelo favoreció el incremento en los stocks de C del suelo (Novelli et al., 2011); por otra parte, Bordin et al., (2008) afirma que los residuos de maíz tienen altos contenidos de lignina con alta relación $\mathrm{C} / \mathrm{N}$, favoreciéndose los procesos de humificación en los suelos.

El cultivo de soya es una leguminosa que tiene un excelente potencial para dinamizar la agricultura sostenible de la Orinoquia (Benavides, 2010); las coberturas de leguminosas son alternativas importantes para incrementar las entradas de $\mathrm{N}$ en suelos tropicales (Friesen et al., 1998); resultando en incrementos en producción de biomasa y acumulación de $\mathrm{C}$ y $\mathrm{N}$ del suelo (White, 1984; Amado et al., 2006).

En los sistemas de rotación mediante la labranza reducida, los contenidos de MOS pueden ser preservados, pues hay una reducción en la tasa de descomposición
(Six et al., 2006) en función de la menor fragmentación de los residuos y de la baja incorporación de residuos (Reicosky y Archer, 2007), con menores pérdidas de $\mathrm{C}$ del suelo en forma de $\mathrm{CO}_{2}$ atmosférico (Six et al., 2006).

El clúster II se caracterizó por presentar un stock de C inicial $\left(\mathrm{SOC}_{0}\right)$ inferior al promedio general y una tasa de pérdidas de $C$ del suelo $(-\Delta C)$ inferior a la totalidad de los sistemas analizados, estuvo conformado por los sistemas de monocultivo altamente gestionados que incluyen laboreo total y baja entrada de residuos con altas tasas de pérdidas de $\mathrm{C}$ del suelo $(-\Delta \mathrm{C})$ actuando como emisores de $\mathrm{CO}_{2}$ a la atmósfera (GEI), agrupando a los sistemas de monocultivos de piña (S8) y arroz (S10) de Villavicencio y monocultivos de piña de Puerto López (S14) (Figura 2).

Los usos intensivos del suelo han conducido a un proceso de degradación de los suelos, generando grandes 


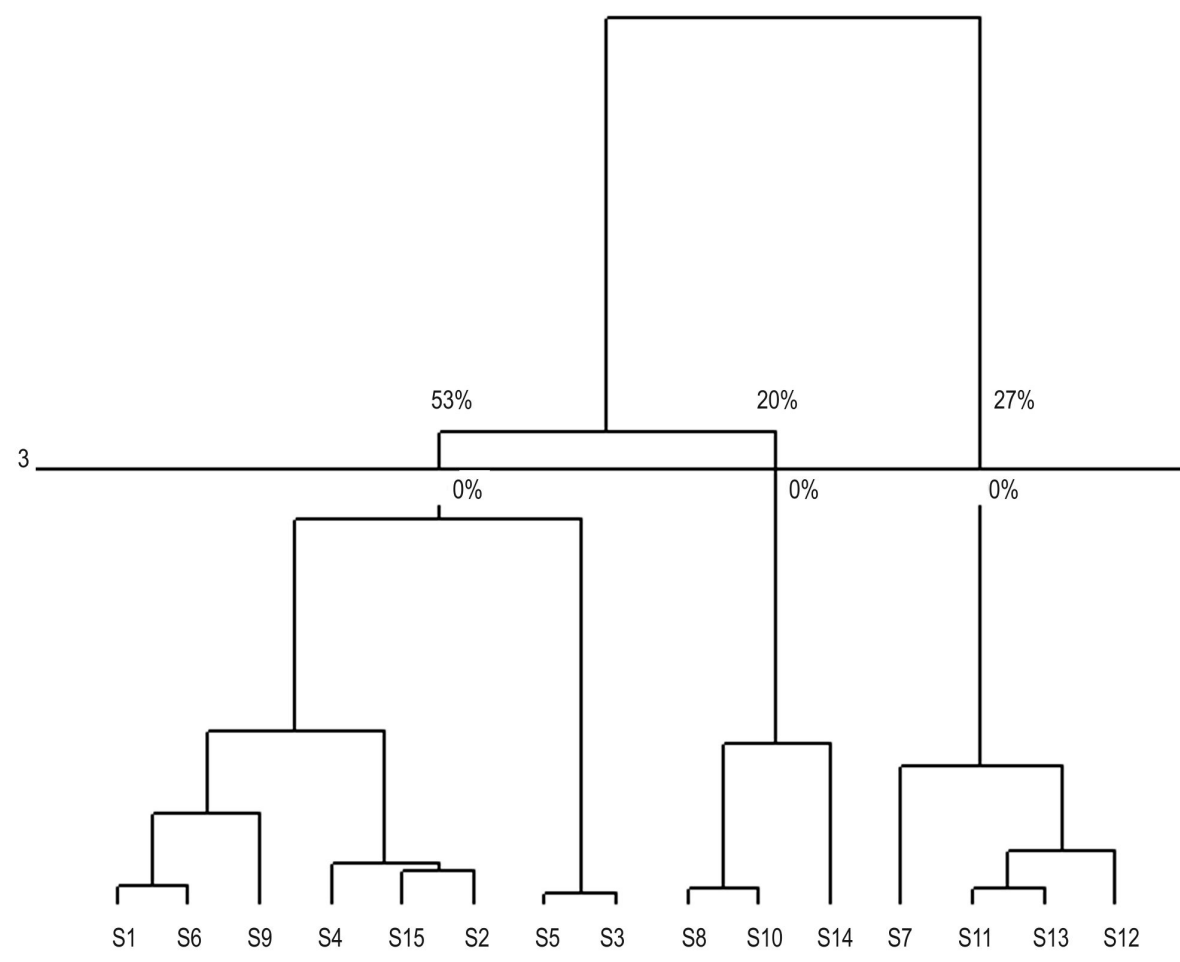

Granada: S1 = Pastura mejorada; S2 = Monocultivo de soya en rotación con maíz; S3 = Cultivo de maíz en rotación con yuca; S4 = Monocultivo de plátano en rotación con yuca; S5 = Monocultivo de arroz en rotación con maíz y soya; Villavicencio: S6 = Pastura mejorada; S7 = SAF de cacao y Acacia mangium; S8 = Monocultivo de piña; S9 = Sistema de café asociado con plátano y leguminosas; S10 = Monocultivo de arroz; Puerto López: S11 = Bosque secundario; S12 = Sistema silvopastoril de Acacia mangium y pastura mejorada; S13 = Sistema agroforestal de caucho y leguminosas de cobertura; S14 = Monocultivo de piña; S15 = Monocultivo de cacao.

Figura 2. Dendograma correspondiente al análisis de agrupamiento de los sistemas productivos analizados con base en los stocks de C y emisiones de GEI a la atmosfera asociados a algunas propiedades fisicoquímicas de los suelos, Altillanura Plana, Meta, Colombia, 2015.

pérdidas en los stocks de COS, por aumento en la oxidación de la MOS y erosión, además de producir otros efectos negativos en el ambiente (Lal, 2008).

En monocultivos donde se ha realizado labranza convencional por muchos años, con intensas remociones de suelo, se ocasiona un rompimiento de los agregados del suelo, exponiendo parte del $\mathrm{C}$ protegido en su interior, dando origen a una mayor oxidación de la MOS, tornándolos susceptibles a una mayor mineralización (Six et al., 2006; Chen et al., 2009; Campos et al., 2012), y por consiguiente, a una disminución de los contenidos de MOS, y pérdidas de C (La Scala et al., 2009; Campos et al., 2011), que se traducen en menores stocks de $\mathrm{C}$ del suelo y mayores emisiones de $\mathrm{CO}_{2}$ atmosférico (Romanenkov et al., 2008).

La conversión de monocultivo de arroz de Villavicencio (S10) para el sistema de monocultivo de arroz en rotación con maíz y soya de Granada (S5) se estaría aumentando el stock de $\mathrm{C}$ en cerca de 12.31 t $\mathrm{C}$ ha en 20 años, equivalente a 0.61 t $C$ ha-1 año ${ }^{-1}$, lo cual reduciría las emisiones en 2.27 t CO 2 eq ha-1 año ${ }^{-1}$.

El clúster III agrupa a los sistemas agroforestales (SAFs) de cacao con Acacia mangium de Villavicencio (S7), con bosque secundario de Puerto López (S11) con un uso de suelo no gestionado, sin laboreo y alta entrada de residuos siendo similares a los sistemas agroforestales de caucho asociados con leguminosas de porte bajo (S13) y los sistemas silvopastoriles de pasturas mejoradas con Acacia mangium (S12) de Puerto López (Figura 2).

Coincidiendo con lo mencionado por Latriglia y Vera $(2014,89)$ "En la zona de la Altillanura Colombiana se ha observado un alto potencial para la recuperación de áreas degradadas, captura y almacenamiento 
de carbono (C), puesto que existen grandes extensiones de sistemas pastoriles, por tanto se podrían implementar proyectos que conlleven a la reducción de emisiones de gases de efecto invernadero (GEI), particularmente de dióxido de carbono $\left(\mathrm{CO}_{2}\right)$, lo cual podría ser similar al de algunos ecosistemas de bosque nativo secundario".

El cambio en uso del suelo de sistemas de monocultivo de cacao de Puerto López (S15) para sistemas agroforestales (SAFs) de cacao con Acacia mangium de Villavicencio (S7) y de pasturas mejoradas de Granada (S1) para sistemas silvopastoriles (SSPs) de pasturas mejoradas con Acacia mangium de Puerto López (S12) incrementarían los stocks de $C$ del suelo en cerca de 17.72 y 40.92 t C ha $^{-1}$ en 20 años, equivalente a 0.88 y $2.04 \mathrm{t}$ $\mathrm{C}$ ha ${ }^{-1}$ año $^{-1}$ y se neutralizarían emisiones de cerca de 3.25 y 7.48 t CO$_{2} \mathrm{eq} \mathrm{ha}^{-1} \mathrm{año}^{-1}$ respectivamente.

Un sistema agroforestal (SAF) de cacao tiene la capacidad de aportar gran cantidad de biomasa y retornarla al suelo, fijando cerca de 3 t C ha ${ }^{-1} \mathrm{año}^{-1}$ con una reducción de 11 t CO 2 eq ha ${ }^{-1}$ año $^{-1}$ (Jadan et al., 2015). Por otra parte, comparando un sistema de pastura de Pennisetum clandestinum sin árboles con un sistema silvopastoril de la misma pastura asociada con Acacia decurrens de 6 años de edad, el almacenamiento de $C$ en el suelo varió de 139.99 a 156.21 y t C ha-1 (Giraldo et al., 2008).

Los esfuerzos generados en la adopción de sistemas silvopastoriles (SPSs) han sido referenciados por varios autores (Amézquita et al., 2004; Nair et al., 2010; Saha et al., 2010); el potencial que tienen estos sistemas en el almacenamiento de $\mathrm{C}$ del suelo ayudan en parte a reducir las emisiones de GEI (Nair et al., 2010).

\section{Conclusión}

En los sistemas agroforestales y silvopastoriles de Puerto López, los stocks de $\mathrm{C}$ y las emisiones de $\mathrm{CO}_{2}$ a la atmosfera mostraron un balance positivo de almacenamiento de $\mathrm{C}$ en el suelo y negativo en los niveles atmosféricos de GEl, que pueden contribuir en parte a mitigar los efectos negativos del cambio climático de la Altillanura Plana de Colombia y de los GEI generados a nivel mundial.

\section{Agradecimientos}

A la Dirección General de Investigaciones y la Facultad de Ciencias Agropecuarias y Recursos Naturales FCAyRN de la Universidad de los Llanos - UNILLANOS, por el apoyo financiero y acompañamiento a esta investigación.
Al Grupo de Investigación en Innovación en Sistemas Agrícolas y Forestales ISAF de la Unillanos, por todas las contribuciones de sus participantes.

\section{Bibliografía}

Amado TJC, Bayer C, Conceicao PC, Spagnollo E, Campos BC, Veiga $M$. Potential of carbon accumulation in no-till soils with intensive use and cover crops in Southern Brazil. J Environ Qual. 2006;35:1599-1607.

Amézquita E, Sanz JI, Thomas RJ, Vera RR, Hoyos P, Molina DL, Chávez LF. Características estructurales de los suelos de los Llanos Orientales de Colombia sometidos a varios sistemas de manejo. Revista Suelos Ecuatoriales. 1997;27:151-156.

Amézquita $E$, Friesen D, Rivera $M$, Rao IM, Barrios E, Jiménez JJ, Decaens T, Thomas RJ. 2002. Sustainability of crop rotation and ley pasture systems on the acid-soil savannas of South America. In: Proceedings of the 17th World Congress of Soil Science, Bangkok, Tailandia, 14-21 Agosto 2002.

Amézquita E. 2013. Propiedades Físicas de los Suelos de los Llanos Orientales y sus Requerimientos de Labranza. En: Sistemas agropastoriles: Un enfoque integrado para el manejo sostenible de Oxisoles de los Llanos Orientales de Colombia /editado por Edgar Amézquita, Idupulapati M. Rao, Mariela Rivera, Irlanda I. Corrales y Jaime H. Bernal. -- Cali, CO: Centro Internacional de Agricultura Tropical (CIAT); Ministerio de Agricultura y Desarrollo Rural (MADR) de Colombia; Corporación Colombiana de Investigación Agropecuaria (Corpoica), 2013. 288 p. -- (Documento de Trabajo CIAT No. 223). ISBN 978-958-694-117-4

Andrade HJ, Figueroa JMP, Silva DP. Carbon storage in cacao (Theobroma cacao) plantations in Armero - Guayabal (Tolima, Colombia). Scientia Agroalimentaria. 2013;1:6-10.

Ávila G, Jiménez F, Beer J, Gómez M, Ibrahim, M. Almacenamiento, fijación de carbono y valoración de servicios ambientales en sistemas agroforestales en Costa Rica. Agrofor Am. 2001;8(30):32-35.

Basamba TA, Barrios E, Amézquita E, Rao IM, Singh BR. Tillage effects on maize yield in a Colombian savanna Oxisol: Soil organic matter and P fractions. Soil Tillage Res. 2006;91(1-2):131142.

Bayer C, Martin-Neto L, Mielniczuk J, Pavinato A, Dieckow J. Carbon sequestration in two Brazilian Cerrado soils under no-till. Soil Tillage Res. 2006;86:237-245.

Benavides J. 2010. El desarrollo económico de la Orinoquia, como aprendizaje y construcción de instituciones. Debates Presidenciales. CAF-Fedesarrollo. 40 p.

Bernoux M, Cerri CC, Cerri CEP, Siqueira Neto M, Metay A, Perrin AS, Scopel E, Razafimbelo T, Blavet D, Piccolo MC, Pavei M and Milne E. Cropping systems, carbon sequestration and erosion in Brazil. Agron Sustain Dev. 2006; 26:1-8.

Boeckx P, Vervaet $\mathrm{H}$, Van Cleemput, O. $\mathrm{NO}$ and $\mathrm{N}_{2} \mathrm{O}$ fluxes from a Belgian forest affected by elevated nitrogen deposition. Guyana Bot. 2005;62:72-87. 
Bordin I, Neves CSVJ, Medina CC, Santos JCF, Torres E, Urquiaga S. Matéria seca, carbono e nitrogênio de raízes de soja e milho em plantio direto e convencional. Pesq Agropec Bras. 2008;12:1785-1792.

Brady NC, Weil RR. 2002. The nature and properties of soils. 13th ed. New Jersey, United States of America, Prentice Hall, 498$542 \mathrm{p}$.

Campos BC, Amado TJC, Bayer C, Nicoloso R, Fiorin JE. Carbon stock and its compartments in a subtropical Oxisol under long-term tillage and crop rotation systems. R Bras Ci Solo. 2011;35:805-817.

Castillo J, Amézquita E, Muller-Samann K. La turbidimetría una metodología promisoria para caracterizar la estabilidad estructural de los suelos. Suelos Ecuatoriales. 2000;30(2):152-156.

Cerri CEP, Sparovek G, Bernoux M, Easterling WE, Melillo JM, Cerri CC. Tropical agriculture and global warming: impacts and mitigations options. Sci Agric. 2007;64:83-99.

Chen HQ, Hou RX, Gong YS, Li HW, Fan MS, Kuzyakov Y. Effects of 11 years of conservation tillage on soil organic matter fractions in wheat monoculture in Loess Plateau of China. Soil Till Res. 2009;106:85-94

Cochrane TT, Sanchez LG, Azevedo LG, Porras JH, Garver CL. 1985. Land in Tropical America. CIAT, Cali, Colombia and EMBRAPA, Planaltina, D.F., BRAZIL. ISBN 84-89206. 3 vols and maps.

Conant RT, Paustian K, Elliott ET. Grassland Management and Conversion into Grassland: Effects on Soil Carbon. Ecol Appl. 2001;11:343-355.

Dossa E, Fernandes E, Reid W, Ezui K. Above- and below-ground biomass, nutrient and carbon stocks contrasting an open-grown and a shaded coffee plantation. Agrofor Syst. 2008;72:103-115.

Friesen D, Thomas R, Rivera M, Asakawa N, Bowen W. 1998. Nitrogen dynamics under monocultures and crop rotations on a Colombian savanna Oxisol. En: Proceedings of the 16th World Congress of Soil Science on CD-ROM. Association française pour l'étude du sol, Montpellier, Francia.

Giraldo A, Zapata M, Montoya E. Carbon capture and flow in a silvopastoral system of the Colombian Andean zone. Asociación Latinoamericana de Producción Animal. 2008;16(4):241-245.

IGAC - Instituto Geográfico Agustín Codazzi. 2006. Métodos analíticos de laboratorio de suelos. Bogotá, Colombia, IGAC, 8-411.

IPCC - Intergovernmental Panel on Climate Change. 2006. Guidelines for national greenhouse gas inventories. En: Eggleston HS, Buendía HS, Miwa L, Ngara K, Tanabe K. (Ed.). Agriculture, Forestry and Other Land Use. (595 p). Hayama: Nacional Greenhouse Gas Inventories Programme. Institute for Global Environmental Strategies (IGES).

IPCC - Intergovernmental Panel on Climate Change. 2007. Climate change impacts, adaptation and vulnerability. Summary for policy makers. Paris: 2007.

Jadán O, Cifuentes $M$, Torres B, Selesi D, Veintimilla D, Günter S. Influence of tree cover on diversity, carbon sequestration and productivity of cocoa systems in the Ecuadorian Amazon. Bois et forêts des Tropiques. 2015;325(3):35-47.
Jaramillo D. 2002. Introducción a la Ciencia del Suelo. Universidad Nacional de Colombia, Facultad de Ciencias, 613 p.

Kaul M, Dadhwal VK, Mohren GMJ. Land use change and net C flux in Indian forests. Forest Ecol Manag. 2009;258:100-108.

Kirby KR, Potvin C. Variation in carbon storage among tree species: implications for the management of a small-scale carbon sink project. For Ecol Manag. 2007;246:208-221.

Lal R, Follet RF, Kimble J, Cole CV. Managing U.S. cropland to sequester carbon in soil. J Soil Water Conserv. 1999;5:374-381.

Lal R. Managing soils and ecosystems for mitigating anthropogenic carbon emissions and advancing global food security. Bioscience. 2010;60:708-721.

Lal R. Soil carbon sequestration impacts on global climate change and food security. Science. 2004;304:1623-1627.

Lal R. Carbon sequestration. Phil. Trans. R Soc B. 2008;363:815-830.

La Scala N Jr, Marques Jr J, Pereira GT, Corá JE. Carbon dioxide emission related to chemical properties of a tropical bare soil. Soil Biol Biochem. 2000;32:1469-1473.

La Scala N Jr, Lopes A, Spokas K, Archer D, Reicosky DC. Short-term temporal changes of bare soil $\mathrm{CO}_{2}$ fluxes after tillage described by first-order decay models. Eur J Soil Sci. 2009;60:258-264.

Latriglia CLX, Vera OC. Captura de Carbono en sistemas pastoriles establecidos en Colombia. Rev Sist Prod Agroecol. 2014;6(1):89-113.

Liu A, Ma BL, Bomke AA. Effects of cover crops on soil aggregate stability, total organic carbon, and polysaccharides. Soil Sci Soc Am J. 2005;69: 2041-2048.

Lopes AS, Ayarza M, Thomas RJ. 2004. Managing and conserving acid savanna soils for agricultural development: Lessons from the Brazilian Cerrados. En: Guimarães EP, Sanz JI, Rao IM, Amézquita MC, Amézquita $E$, Thomas RJ (eds). Agropastoral systems for the tropical savannas of Latin America. Centro Internacional de Agricultura Tropical (CIAT), Cali, Colombia; Empresa Brasileira de Pesquisa Agropecuária (Embrapa), Brasilia, DF, Brasil. p 11-41.

Lovato T, Mielniczuk J, Bayer C, Vezzani C. Adição de carbono e nitrogênio e sua relação com os estoques no solo e com o rendimento do milho em sistemas de manejo. R Bras Ci Solo. 2004;28:175-187.

Malagón D. Ensayo sobre tipología de suelos colombianos - énfasis en génesis y aspectos ambientales. Revista Acad Colomb Ci Exact. 2003;27(104):319-341.

Manly BFJ. 1997. Multivariate statistical methods. A primer. Second Ed. Chapman \& Hall, London. 216 p.

Nair PKR, Nair VD, Kumar BM, Showalter JM. Carbon sequestration in agroforestry systems. Adv Agron. 2010;108:237-307.

Novelli LE, Caviglia OP, Melchiori RJM. Impact of soybean cropping frequency on soil carbon storage in Mollisols and Vertisols. Geoderma. 2011;167-168: 254-260. 
Ogle SM, Breidt FJ, Paustian K. Agricultural management impacts on soil organic carbon storage under moist and dry climatic conditions of temperate and tropical regions. Biogeochemistry. 2005;72:87-121.

Reeves DW. The role of soil organic matter in maintaining soil quality in continuous cropping systems. Soil Till Res. 1997;43:131-167.

Reicosky DC, Archer DW. Moldboard plow tillage depth and shortterm carbon dioxide release. Soil Till Res. 2007;94:109-121.

Romanenkov V, Schneider U, Towprayoon S, Wattenbach M, Smith J. Greenhouse gas mitigation in agriculture. Phil Trans R Soc B. 2008;363:789-813.

Saha SK, Nair PKR, Nair VD, Kumar BM. Carbon storage in relation to soil size-fractions under some tropical tree-based land-use systems. Plant Soil. 2010;328:433-446
Six J, Frey SD, Thies RK, Batten KM. Bacterial and fungal contributions to carbon sequestration in agroecosystems. Soil Sci Soc Am J. 2006;70:555-569.

Somarriba E, Cerda R, Orozco L, Cifuentes M, Davila H, Espin T. et al. Carbon stocks and cocoa yields in agroforestry systems of Central America. Agric Ecosyst Environ. 2013;173:46-57.

Yoon S, Wattenbach M, Smith J. Greenhouse gas mitigation in agriculture. Phil Trans. R Soc B. 2008;363:789-813.

White PJ. Effects of crop residues incorporation on soil properties and growth of subsequent crops. Aust J Exp Agric Anim Husb. $1984 ; 24: 219-235$. 